\title{
Effect of panretinal photocoagulation on macular morphology and thickness in eyes with proliferative diabetic retinopathy without clinically significant macular edema
}

\author{
This article was published in the following Dove Press journal: \\ Clinical Ophthalmology \\ 3 December 2012 \\ Number of times this article has been viewed
}

\author{
Manoj Soman' \\ Sunil Ganekal² \\ Unnikrishnan Nair' \\ KGR Nair' \\ 'Chaithanya Eye Hospital and \\ Research Institute, Trivandrum, \\ Kerala, India; ${ }^{2}$ Nayana Superspecialty \\ Eye Hospital and Research Center, \\ Davangere, Karnataka, India
}

\begin{abstract}
Background: The purpose of this study was to investigate the effect of panretinal photocoagulation (PRP) on macular morphology and thickness in eyes with proliferative diabetic retinopathy (PDR) and without clinically significant macular edema.

Methods: This was a prospective study of 76 eyes from 68 patients diagnosed to have PDR without clinically significant macular edema. Baseline and post PRP visual acuity, morphological changes on optical coherence tomography (OCT), and central foveal thickness were evaluated at one week, one month, and 3 months.

Results: The mean patient age was $56.47 \pm 6.55$ years. Sixty-two eyes $(81.58 \%)$ had stable or improved vision, while 14 eyes $(18.42 \%)$ had worsened visual acuity at 3 months. Compared with baseline, mean visual acuity dropped as early as one week but was regained by 3 months. Mean preoperative central foveal thickness was $222.05 \pm 59.11 \mu \mathrm{m}$, which increased significantly to $266.84 \pm 84.67 \mu \mathrm{m}$ at one week $(P=0.001)$, and remained higher at $264.05 \pm 102.56 \mu \mathrm{m}$ by one month $(P=0.01)$ and $256 \pm 101.38 \mu \mathrm{m}$ by 3 months $(P=0.04)$. Thirty-four percent of eyes with a normal macula showed morphological changes following PRP. The most common morphological change on OCT after PRP was spongy edema, seen in 48 eyes (31.6\%), followed by cystoid macular edema in 36 eyes (23.7\%), vitreomacular traction in 28 eyes (18.4\%), epiretinal membrane in 24 eyes (15.8\%), and subfoveal serous detachment in 16 eyes (10.5\%).

Conclusion: PRP may cause a temporary drop in vision in the early post laser phase, and causes macular morphology/thickness changes in eyes with PDR and without clinically significant macular edema. In this study, the change in central foveal thickness did not correlate with a change in visual acuity, and the type of diabetic macular edema on OCT appeared more relevant and correlated better with the visual outcome.
\end{abstract}

Keywords: panretinal photocoagulation, macular morphology, foveal thickness

\section{Introduction}

Macular edema is the most common cause of visual loss in patients with proliferative diabetic retinopathy (PDR) and in those with nonproliferative diabetic retinopathy. ${ }^{1}$ Panretinal photocoagulation (PRP) appears to be beneficial for patients with PDR, as shown by the Diabetic Retinopathy Study and the Early Treatment Diabetic Retinopathy Study. ${ }^{2,3}$ In some cases, visual acuity decreases after PRP, but usually improves over several weeks. Macular edema and serous detachment of the macula has been observed after PRP, but it is not clear whether this is related to photocoagulation or if it is a coincidental finding. ${ }^{4-6}$ Studies have shown that $25 \%-43 \%$ 
of eyes with PDR treated using PRP develop increased macular edema and visual disturbances. ${ }^{5-7}$ Studies have also shown that PRP in eyes with nonproliferative diabetic retinopathy and early PDR affects visual field sensitivity, but not visual acuity. ${ }^{5,7,8}$ It is also known that patients with clinically significant macular edema worsen after having PRP for coexisting PDR. ${ }^{5,6}$ Optical coherence tomography (OCT) is an imaging modality that currently helps in both the quantitative ${ }^{9}$ and qualitative ${ }^{10}$ study of macular edema and its characteristics. Although fluorescein angiography is typically used to assess vascular leakage qualitatively in patients with macular edema, assessment of actual macular thickening correlates better with loss of visual acuity. Traditional methods of evaluating macular thickening, including slit-lamp examination and stereo fundus photography, are relatively insensitive to small changes in retinal thickness. OCT has proved to be a sensitive tool for detecting subtle cystoid macular edema and subretinal fluid, which may be early and severe manifestations of macular edema and not visualized on clinical ${ }^{11}$ or angiographic ${ }^{12,13}$ analysis. Vitreomacular traction is another entity which can be studied effectively on OCT and is usually missed on routine clinical evaluation. ${ }^{14}$ These macular changes may be the cause of visual deficits in patients after PRP. Although the mechanism of how macular edema develops after PRP remains unclear and various postulates are available, increased foveal and parafoveal thickening may be a cause of visual dysfunction in these patients. ${ }^{2,15}$ Thus, there may be some hesitation in performing PRP in diabetic patients with proliferative disease, especially in eyes with no macular edema and good visual acuity, and questions arise regarding which systemic factors or conditions in the retina can predict visual outcome after PRP and the probability of visual dysfunction induced by PRP. This study was undertaken to investigate the clinical and tomographic correlation between visual changes and macular morphology in patients undergoing PRP for PDR without clinically significant macular edema.

\section{Materials and methods}

This was a prospective study of 76 eyes from 68 diabetic patients attending our retina clinic over a period of 6 months. The study was approved by our institutional review board. Inclusion criteria at baseline were early PDR (without high-risk characteristics) without clinically significant macular edema. Early PDR was identified by the presence of new vessels on $\leq$ one-third of the disc diameter without any preretinal subhyaloid or vitreous hemorrhage or new vessels elsewhere in the retina. Eyes with clinically significant macular edema were excluded, along with patients who had had cataract surgery and/or focal laser treatment within the previous 3 months. The presence of corneal opacity, cataract, uveitis, glaucoma, aphakia, and poor visual acuity due to any other cause were also grounds for exclusion.

Examination included assessment of visual acuity using a Snellen chart, anterior segment examination by slit-lamp biomicroscopy, intraocular pressure measurement with applanation tonometry, and fundus examination by slitlamp biomicroscopy and indirect ophthalmoscopy. Vision was recorded using the Snellen chart and converted to the logarithm of the minimum angle of resolution ( $\log M A R)$ for data analysis. Fundus photography was done for documentation and follow-up whenever necessary. Fundus fluorescein angiography and OCT were carried out for all patients before PRP. Fluorescein angiography was done to rule out macular ischemia and to confirm early PDR. Patients with macular ischemia were excluded from the study at baseline because this could have had a bearing on the visual outcome and be a confounding variable during analysis. OCT was done before and one week, one month, and 3 months after PRP. Line scan and fast macular scans were done to study the macula on the Stratus OCT (Carl Zeiss Meditec, Dublin, CA, USA), and a 5 -line raster scan and cube $512 \times 200$ scan was done on the Cirrus OCT (Carl Zeiss Meditec, Dublin, CA, USA). The line scan/5-line raster protocol were used to study the morphological features of macular edema, ie, spongy edema/ spongy retinal thickening, epiretinal membrane, vitreomacular traction, subretinal fluid, and cystoid macular edema. Spongy edema/retinal thickening was defined as increased retinal thickness with reduced intraretinal reflectivity and expanded areas of lower reflectivity on OCT. Fast macular protocol/cube data were used to study central foveal thickness. PRP was done with standard parameters using green laser in 3-4 sittings, with a one-week interval between each sitting. After completion of PRP, patients were followed up at one week, one month, and 3 months. At each visit, visual acuity was assessed by Snellen chart, and a qualitative and quantitative study of the macula was done with OCT and clinical examination. Comparison of visual acuity and macular changes with regard to morphological and macular thickness was made at each post treatment visit. The Chisquare test, paired $t$-test, and Pearson's correlation were used in the statistical analysis, which was performed using the Statistical Package for the Social Sciences version 19.0 (SPSS Inc, Chicago, IL). 


\section{Results}

The mean age of the patients was $56.47 \pm 6.55$ (range 45-69) years. There were 38 males and 30 females, and the mean duration of diabetes was 17.13 (range 10-25) years. Thirty-six of the 68 patients (47.4\%) were on oral hypoglycemic agents for diabetes, 27 (35.5\%) were on insulin, and five $(6.6 \%)$ were on both types of agents. Concomitant systemic diseases included hypertension in 39 patients $(57.4 \%)$, dyslipidemia in 12 (17.7\%), nephropathy in $12(17.7 \%)$, and cardiac disease in $14(20.6 \%)$.

Sixty-two of the 76 eyes $(81.58 \%$ ) had stable or improved vision, while 14 eyes $(18.42 \%)$ had worsened by 3 months following PRP (Table 1). Patients who had improved or stable vision were older $(56.77 \pm 7.07$ years) than those who had worse vision at the end of follow-up ( $55.14 \pm 3.58$ years). Gender, duration of diabetes, and initial visual acuity were not significant influencing factors with respect to final visual outcome. Mean preoperative vision was $0.15 \pm 0.12 \log$ units, which worsened significantly to $0.31 \pm 0.20 \log$ units $(P=0.001)$ at one week, to $0.29 \pm 0.23 \log$ units at one month $(P=0.0001)$, and although worsened at 3 months to $0.25 \pm 0.37 \log$ units, this was not statistically significant $(P=0.06$, Figure 1$)$.

Mean preoperative central foveal thickness was $222.05 \pm 59.11 \mu \mathrm{m}$, increasing to $266.84 \pm 84.67 \mu \mathrm{m}$ at one week, to $264.05 \pm 102.56 \mu \mathrm{m}$ at one month, and to $256 \pm 101.38 \mu \mathrm{m}$ at 3 months' follow-up after PRP (Figure 2). Central foveal thickness was significantly increased from the pretreatment value at one week $(P=0.001)$ and one month $(P=0.01)$, and remained so at 3 months' follow-up $(P=0.04)$. Degree of central foveal thickness at baseline did not correlate with the final visual outcome. However, there was a positive correlation between change in central foveal thickness and visual acuity at one week, one month, and 3 months after PRP.

OCT features at baseline were mainly normal (54 of 76 eyes, $71.7 \%$ ). The most common abnormality seen was spongy edema in 14 eyes, (18.4\%), followed by epiretinal membrane in four eyes $(5.3 \%)$, and cystoid macular edema, subretinal fluid, and vitreomacular traction in two eyes each (2.6\%). Three months after PRP, only 28 eyes $(36.8 \%)$ had normal OCT. The most common abnormality was spongy

Table I Visual acuity changes following panretinal photocoagulation

\begin{tabular}{lll}
\hline Vision & $\mathbf{n}$ & $\%$ \\
\hline Stable or improved & 62 & 81.58 \\
Worsened & 14 & 18.42 \\
Total & 76 & 100 \\
\hline
\end{tabular}

Abbreviation: $\mathrm{n}$, number of eyes.

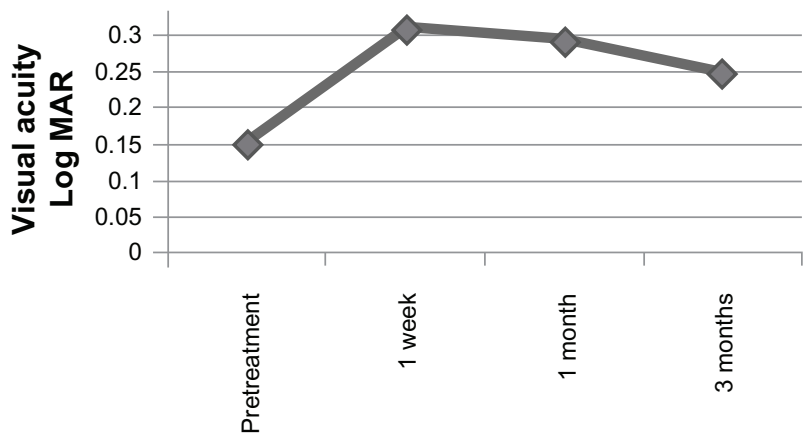

Figure I Mean change in visual acuity over 3 months.

edema, seen in 24 eyes (31.6\%), followed by cystoid macular edema in 18 eyes (23.7\%), vitreomacular traction in 14 eyes (18.4\%), epiretinal membrane in 12 eyes $(15.8 \%)$, and subfoveal subretinal fluid in eight eyes $(10.5 \%)$. All these features were more common following treatment than at baseline (Figure 3). However, correlation with visual outcome revealed that patients in the group with worsened vision had significantly more spongy edema $(P=0.05)$, vitreomacular traction $(P=0.013)$, and subretinal fluid $(P=0.001)$. The increased occurrence of cystoid macular edema or epiretinal membrane following PRP did not seem to affect the visual outcome.

\section{Discussion}

The mean age of our study population was $56.47 \pm 6.55$ years, and all the patients were type 2 diabetics with a mean duration of diabetes of 17 years. Most of the patients (81.58\%) had stable or improved vision 3 months after PRP. Patients who had improved or stable vision were older $(56.77 \pm 7.07$ years) than those who had worsened vision ( $55.14 \pm 3.58$ years), but this difference was not statistically significant. The Wisconsin Epidemiological Study of Diabetic Retinopathy also found that the severity of diabetic retinopathy and clinically significant macular edema is not related to age, but is related to duration of diabetes. Gender and duration of diabetes were not significant

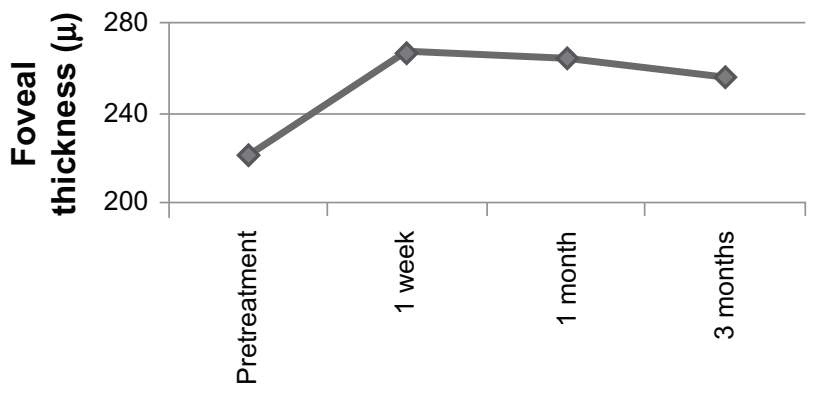

Figure 2 Mean change in central foveal thickness over 3 months. 


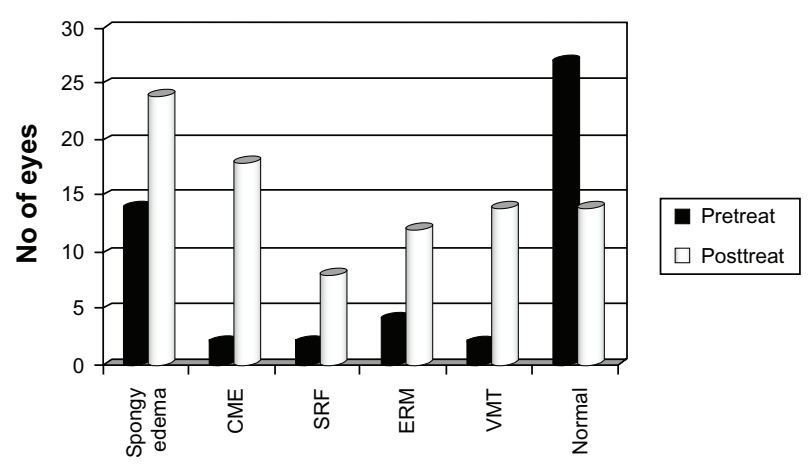

Figure 3 Change in macular morphology on optical coherence tomography before and after panretinal photocoagulation.

Abbreviations: CME, cystoid macular edema; SRF, subretinal fluid; ERM, epiretinal membrane; VMT, vitreomacular traction.

influencing factors with respect to final visual outcome. These observations indicate that the final visual acuity is governed by laser treatment and any change in macular thickness.

In our study, $81.58 \%$ of patients had stable or improved vision, while $18.42 \%$ had worse vision 3 months after PRP. McDonald et $\mathrm{al}^{4,5}$ reported that $25 \%$ of eyes treated with PRP lost $\geq 2$ lines after a mean follow-up of 15 months. The Diabetic Retinopathy Study reported $10 \%$ of eyes with visual loss after PRP. Overall, 25\%-43\% of eyes have been reported to have visual loss or changes after PRP in various studies. ${ }^{4,5}$ However, many of these studies also included eyes with preexisting macular edema. Shimura et $\mathrm{al}^{15}$ reported that in eyes with PDR and without clinically significant macular edema, PRP did not affect postoperative vision in $85 \%$ of eyes, which is comparable with the findings of our study $(82 \%)$. PRP inducing visual loss in these eyes was less common, accounting for $15 \%$ of eyes, as in our study (18\%). Thus, eyes with PDR and without clinically significant macular edema generally tolerate the insult to the blood-retinal barrier during PRP reasonably well. Patients in this subgroup who lose vision probably have other systemic or local contributing factors that precipitated macular edema.

Associated systemic diseases, eg, hyperlipidemia, diabetic nephropathy, carotid occlusive disease, and anemia, as well as local factors, eg, post cataract status and coexisting uveitis, have been reported to cause a greater exudative response following PRP, and so more macular edema and visual loss. In the present series, all of the 14 eyes with decreased vision following PRP were from patients who had multiple systemic diseases, including hypertension (100\%), nephropathy (71\%), cardiac disease (29\%), and dyslipidemia (29\%). The commonest cause of defective vision was macular edema, seen in $86 \%$ (12 of 14 eyes) of this group. Eyes which previously had no macular pathology now had cystoid macular edema (43\%), subretinal fluid (57\%), epiretinal membrane (29\%), or vitreomacular traction (57\%) after PRP. The remaining two eyes developed vitreous hemorrhage as the cause of defective vision.

Defective vision following PRP was also studied by McDonald et al, ${ }^{5}$ who found that the main causes were macular edema (32\%) followed by vitreous hemorrhage (23\%), tractional retinal detachment (14\%), epiretinal membrane (9\%), macular ischemia (7\%), cataract, and neovascular glaucoma (5\%). In the present study, two eyes (2.6\%) developed significant vitreous hemorrhage, but none developed tractional retinal detachment or neovascular glaucoma.

After PRP, mean vision decreased at one week and one month, but stabilized by 3 months. Even though $18 \%$ of patients had visual loss by the end of 3 months of followup, mean vision in this group did not decrease significantly from baseline. Shimura et $\mathrm{al}^{15}$ reported that $84 \%$ of their patients with PDR and without clinically significant macular edema maintained vision during follow-up after PRP, but that visual acuity dropped over $0-8$ weeks after PRP in 5\% of eyes, which later regained their baseline values. In $11 \%$ of eyes, visual acuity had dropped by 2 weeks after PRP and continued to decrease during follow-up. The cause of visual loss in the latter two groups was macular edema, as was seen in our series as well.

It has been reported that the average foveal thickness in normal subjects is approximately $178 \mu \mathrm{m}$, and in contrast, the average foveal thickness in eyes with clinically significant macular edema is $362-572 \mu \mathrm{m}$, which is at least twice that in normal subjects. Studies have also shown that average foveal thickness is greater than normal in patients with diabetes and no retinopathy and in patients with retinopathy and no macular edema. ${ }^{16,17}$ In our study, the mean pretreatment central foveal thickness was $222 \mu \mathrm{m}$, which increased during follow-up and remained higher at $256 \mu \mathrm{m}$ (an 87\% increase) by 3 months follow-up. This increase was seen as early as one week following treatment. Although there was a generally positive correlation between the change in foveal thickness and the change in visual acuity, the change in central foveal thickness did not correlate with the change in visual acuity. Mean central foveal thickness at final follow-up remained high, although the mean visual acuity had normalized by the end of 3 months of follow-up. Similar observations were reported by Shimura et $\mathrm{al}^{18}$ in eyes that underwent PRP sessions on a weekly basis than those who underwent PRP biweekly. They found that visual acuity was unaltered in spite of a $142 \%$ increase in mean foveal thickness, and the type of 
macular edema seen on OCT appeared to be more relevant and better correlated with visual outcome than the quantitative estimation of foveal thickness. In our study, eyes with subretinal fluid and vitreomacular traction had a worse visual outcome than those with cystoid macular edema and epiretinal membrane. These eyes may require additional therapy, as observed by some authors who have found that even in eyes with PDR undergoing PRP, posterior sub-Tenon's steroid injection has beneficial effects for preventing PRP-induced foveal thickening and visual dysfunction. Various authors have reported poor visual outcomes in diabetic eyes with cystoid macular edema, subretinal fluid, and vitreomacular traction, which supports our observations. ${ }^{12-14}$ Thus, foveal thickness estimation may not be an appropriate guide as to visual status. The Diabetic Retinopathy Clinical Research Network ${ }^{19}$ has reported that foveal thickness on OCT does not correlate with visual acuity, particularly in eyes with macular edema undergoing focal laser treatment, and even shows paradoxical responses. Characteristics of macular edema correlate better with visual outcome, as was seen in this study, where presence of cystoid macular edema and epiretinal membrane resulted in poor visual outcome in this group of patients.

In conclusion, PRP may cause a temporary drop in vision in the early post laser phase. This may be seen as early as one week after PRP and can normalize by 3 months. Macular edema is the commonest cause of this drop in vision. Various changes in macular morphology/thickness occur in these eyes, the most common being cystoid macular edema. Therefore, it is prudent to warn patients of this potential outcome. Eyes which lose vision due to macular edema may need alternative therapy in the form of intravitreal pharmacotherapy or laser. This study also reaffirms the fact that central foveal thickness does not always influence the visual outcome and may show no correlation at all.

\section{Disclosure}

The authors report no conflicts of interest in this work.

\section{References}

1. Klein R, Klein BEK, Moss SE. Visual impairment in diabetes. Ophthalmology. 1984;91:1-9.

Clinical Ophthalmology

\section{Publish your work in this journal}

Clinical Ophthalmology is an international, peer-reviewed journal covering all subspecialties within ophthalmology. Key topics include: Optometry; Visual science; Pharmacology and drug therapy in eye diseases; Basic Sciences; Primary and Secondary eye care; Patient Safety and Quality of Care Improvements. This journal is indexed on Submit your manuscript here: http://www.dovepress.com/clinical-ophthalmology-journal
2. Diabetic Retinopathy Study Research Group. Photocoagulation treatment of proliferative diabetic retinopathy: the second report of DRS findings. Ophthalmology. 1978;85:82-106.

3. Early Treatment Diabetic Retinopathy Study Research Group. Grading diabetic retinopathy from color fundus photograph - an extension of the modified Airlie House Classification. ETDRS Report 10. Ophthalmology. 1991;98:786-790.

4. McDonald HR, Schatz H. Macular edema following panretinal photocoagulation. Retina. 1985;5:5-10.

5. McDonald HR, Schatz H. Visual loss after panretinal photocoagulation for proliferative diabetic retinopathy. Ophthalmology. 1985;92: 388-393.

6. Meyers SM. Macular edema after scatter laser photocoagulation for proliferative diabetic retinopathy. Am J Ophthalmol. 1980;90: 210-216.

7. Higgins KE, Meyers SM, Jaffe MJ, et al. Temporary loss of foveal contrast sensitivity associated with PRP. Arch Ophthalmol. 1986;104: 997-1003.

8. Henriesson M, Heijal A. The effect of PRP on visual acuity, visual fields and on subjective visual impairment in pre-proliferative and early proliferative diabetic retinopathy. Acta Ophthalmol. 1994;72:570-575.

9. Hee MR, Carmen A, Wong C, et al. Quantitative assessment of macular edema with optical coherence tomography. Arch Ophthalmol. 1995;113:1019-1029.

10. Panozzo G, Parolini B, Gusson E, et al. Diabetic macular edema: an OCT based classification. Semin Ophthalmol. 2004;19:13-20.

11. Browning DJ, McOwen MD, Bowen RM, O'Marah TL. Comparison of the clinical diagnosis of diabetic macular edema with diagnosis by optical coherence tomography. Ophthalmology. 2004;111:712-715.

12. Ozdek SC, Erdinç MA, Gürelik G, Aydin B, Bahçeci U, Hasanreisoğlu B. Optical coherence tomographic assessment of diabetic macular edema; comparison with fluorescein angiographic and clinical findings. Opthalmologica. 2005;219:86-92.

13. Ozdemir H, Karacoriu M, Karacoriu S. Serous macular detachment in diabetic cystoid macular edema. Acta Ophthalmol Scand. 2005;83: 63-66.

14. Kim BY, Smith SD, Kaiser PK. Optical coherence tomographic patterns of diabetic macular edema. Am J Ophthalmology. 2006;143:405-412.

15. Shimura M, Yasuda K, Nakazawa T, Tamai M. Visual dysfunction after panretinal photocoagulation in patients with severe diabetic retinopathy and good vision. Am J Ophthalmol. 2005;140:8-15.

16. Hee MR, Puliafito CA, Duker JS, et al. Topography of diabetic macular edema with optical coherence tomography. Ophthalmology. 1998;105:360-370.

17. Yang CS, Cheng CY, Lee FL, Hsu WM, Liu JH. Quantitative assessment of retinal thickness in diabetic patients with and without clinically significant macular edema using optical coherence tomography. Acta Ophthalmol Scand. 2001;79:66-70.

18. Shimura M, Yasuda K, Nakazawa T, Kano T, Ohta S, Tamai M. Quantifying alterations of macular thickness before and after panretinal photocoagulation in patients with severe diabetic retinopathy and good vision. Ophthalmology. 2003;110:2386-2394.

19. Beck RW, Edwards AR, Aiello LP, et al; Diabetic Retinopathy Clinical Research Network (DRCR.net). Three-year follow-up of a randomized trial comparing focal/grid photocoagulation and intravitreal triamcinolone for diabetic macular edema. Arch Ophthalmol. 2009;127:245-251.

\section{Dovepress}

PubMed Central and CAS, and is the official journal of The Society of Clinical Ophthalmology (SCO). The manuscript management system is completely online and includes a very quick and fair peer-review system, which is all easy to use. Visit http://www.dovepress.com/ testimonials.php to read real quotes from published authors. 